\title{
A Novel Statistical Detector for Contourlet Domain Image Watermarking Using 2D-GARCH Model
}

\author{
Maryam Amirmazlaghani $\left.{ }^{(}\right)$ \\ Computer Engineering and IT Department, Amirkabir University of Technology, \\ Tehran, Iran \\ mazlaghani@aut.ac.ir
}

\begin{abstract}
In this paper, we propose a novel watermark detector in contourlet domain using likelihood ratio test (LRT). Since the accuracy of an LRT based watermark detector is dependent on the efficiency of the applied statistical model, first, we study the statistical properties of the contourlet coefficients. Using different tests, we demonstrate that the marginal distribution of contourlet coefficients is heavy-tailed and heteroscedasticity exists in these coefficients. All of the previously proposed models for contourlet coefficients assume that these coefficients are identically distributed, so they can not capture the characteristics of the contourlet coefficients. To overcome this problem, we propose using two dimensional generalized autoregressive conditional heteroscedasticity (2D-GARCH) model for contourlet coefficients that provides an efficient structure for the dependencies of these coefficients. Based on using 2D-GARCH model, a novel LRT based heteroscedastic watermark detector is designed in contourlet domain. Experimental results confirm the efficiency of the proposed watermark detector under different types of attacks and its outperformance compared with alternative watermarking methods.
\end{abstract}

Keywords: Image watermarking $\cdot$ Contourlet transform $\cdot$ Likelihood ratio test $\cdot 2 \mathrm{D}-\mathrm{GARCH}$ model

\section{Introduction}

Digital watermarking which embeds information into an original media by modifying its content has been used in many applications such as copyright protection, broadcast monitoring, content authentication, and covert communication [1]. Distribution of digital data on the internet increases the importance of the intellectual property rights, and copyright protection. In this paper, we focus on image watermarking for copyright protection. Imperceptibility and robustness are two major requirements for copyright protection watermarking approaches. In copyright protection, the main goal is to distinguish whether a specific watermark is present in the received media or not. So, to check the validity of the 
watermark for copyright protection, the detection of the watermark is sufficient [2-4]. In some other applications, the watermark decoding may be required, i.e., the hidden message (watermark) should be extracted and decoded $[5,6]$.

A watermarking scheme consists of two main steps: watermark embedding and watermark detection. According to the embedding method, watermarking techniques can be classified into two categories: spread spectrum based [2$4,7]$ and quantization based techniques [8]. Spread spectrum watermarking provides high level of security and robustness because of spreading the watermark throughout the spectrum of the image. So, it is more popular. In spread spectrum schemes, usually the watermark is embedded in a transformed domain such as the discrete cosine transform (DCT) [9], the discrete wavelet transform (DWT) $[3,7]$ and the contourlet transform [2-4].

Contourlet transform which is an efficient extension of the wavelet transform can capture the smooth contours of images using a double filter bank. Contourlet transform consists of two main steps: Applying Laplacian pyramid to capture point discontinuities and using directional filter bank to obtain directional information [10]. This transform provides some good properties such as multiresolution, nearly critical sampling, and directionality [10]. Moreover, contourlet transform has spreading property that is important in watermarking. It means that inserting the watermark into a special subband causes in spreading out it in all subbands at the step of reconstracting the watermarked image [4].

In the recent years, many contourlet domain image watermarking methods have been proposed $[2,4,6,11,12]$. Using correlation detector is a common way to detect transform domain watermarks $[11,12]$. But, this detector is optimal only when the distribution of data is Gaussian. But, the non-Gaussian nature of contourlet coefficients has been studied in many works such as $[2,4,13]$. So, in this situation, watermark detection can be formulated as a binary hypothesis test and optimal watermark detector can be achieved using Bayesian log-likelihood ratio test (LRT). To design a contourlet domain LRT based watermark detector, the contourlet coefficients should be modeled statistically. More accurate statistical model for contourlet coefficients results in more efficient watermark detector. In the literature, to derive contourlet domain watermark detectors, different statistical models have been proposed such as generalized Gaussian (GG) [14], Bessel K form [2], alpha-stable [4], and Normal Inverse Gaussian (NIG) [6].

In this paper, we propose a novel contourlet domain watermark detector. We embed the watermark in contourlet domain using an additive spread spectrum approach. Then, to design an efficient LRT based watermark detector, first, we study the statistical properties of the contourlet coefficients. All of the previously proposed models for contourlet coefficients suppose that these coefficients are identically distributed. In this work, we demonstrate that heteroscedasticity exists in contourlet coefficients and they are not identically distributed. In this way, we propose to apply a heteroscedastic model called two dimensional generalized autoregressive conditional heteroscedasticity (2D-GARCH) model for the contourlet coefficients. 2D-GARCH model is the two dimensional extension of GARCH model [15] and GARCH model has been introduced for financial 
time series by Bollersrev in [16]. Moreover, we demonstrate the efficiency of 2DGARCH model in capturing the heteroscedasicity of contourlet coefficients and the dependencies among them. Then, we design an LRT based contourlet domain watermark detector using 2D-GARCH model. Experimental results confirm the high efficiency of the proposed detector and its robustness under different kinds of attacks. Also, we compare our proposed watermark detector with some alternative detectors and show the outperformance of our method.

This paper is organized as follows. In Sect. 2, we review the contourlet transform, 2D-GARCH model and discuss the compatibility of contourlet coefficients and 2D-GARCH model. Watermark embedding process and 2D-GARCH based watermark detector are explained in Sect.3. Section 4 reports the simulation results. Finally, Sect. 5 concludes the paper.

\section{Statistical Modeling of Contourlet Coefficients}

In this section, first, we review the contourlet transform and the 2D-GARCH model. Then, we verify the efficiency of $2 \mathrm{D}-\mathrm{GARCH}$ to model the contourlet coefficients.

\subsection{Contourlet Transform}

Wavelet transform can not recognize the smoothness of the images contours. To overcome this problem, many directional image representations have been proposed recently. Contourlet is one of them proposed in [10] that provides an efficient representation for typical images with smooth contours. Contourlet transform of an image constructed in two steps. First, Laplacian pyramid filter is applied that results in subband decomposition. Then, the directional filter bank is used. Combining these two steps makes Pyramidal Directional Filter Bank (PDFB). Contourlet transform is computationally efficient because of using iterated filter banks. It can provide a desired number of direction in each subband and also it is close to critically sampled [10]. So, we use contourlet transform.

\subsection{D-GARCH Model}

Suppose $x(i, j)$ represents a two-dimensional zero mean stochastic process. $x(i, j)$ follows a 2D-GARCH $\left(p_{1}, p_{2}, q_{1}, q_{2}\right)$ model, where $\left(p_{1}, p_{2}, q_{1}, q_{2}\right)$ denotes the order of the model, if

$$
\begin{aligned}
& x(i, j)=\sqrt{h(i, j)} \varepsilon(i, j) \\
& h(i, j)=\alpha_{0}+\sum_{k \ell \in \Lambda_{1}} \alpha_{k \ell} x(i-k, j-\ell)^{2}+\sum_{k \ell \in \Lambda_{2}} \beta_{k \ell} h(i-k, j-\ell),
\end{aligned}
$$

where

$$
\begin{aligned}
& \Lambda_{1}=\left\{k \ell \mid 0 \leqslant k \leqslant q_{1}, 0 \leqslant \ell \leqslant q_{2},(k \ell) \neq(0,0)\right\} \\
& \Lambda_{2}=\left\{k \ell \mid 0 \leqslant k \leqslant p_{1}, 0 \leqslant \ell \leqslant p_{2},(k \ell) \neq(0,0)\right\}
\end{aligned}
$$


$\varepsilon(i, j) \sim \mathcal{N}(0,1)$ is an iid two dimensional random process, and $\left\{\alpha_{0}, \alpha_{01}, \cdots\right.$, $\left.\alpha_{q_{1} q_{2}}, \beta_{01}, \cdots, \beta_{p_{1} p_{2}}\right\}$ are the parameters of model. It is clear that $h(i, j)$ is the conditional variance of $x(i, j)$, therefore $x(i, j)$ is conditionally distributed as

$$
x(i, j) \mid \psi(i, j) \sim \mathcal{N}(0, h(i, j))
$$

where

$$
\psi(i, j)=\left\{\{x(i-k, j-\ell)\}_{k, \ell \in \Lambda_{1}},\{h(i-k, j-\ell)\}_{k, \ell \in \Lambda_{2}}\right\} .
$$

To compute the parameters of model, maximum likelihood estimation (MLE) can be used $[15,17]$.

\subsection{D-GARCH Model for Contourlet Coefficients}

Here, we check the suitability of 2D-GARCH model for contourlet coefficients. Since 2D-GARCH is a heteroscedastic model, first of all, we should study the heteroscedasticity of the contourlet coefficients and their match with the specific type of dependency provided by 2D-GARCH model. In this way, we use the Lagrange-multiplier test proposed in [18] which examines the existence of two dimensional heteroscedasticity and two-dimensional GARCH effect. The results of this hypothesis test are reported by (1) a Boolean decision variable " $\mathrm{H}$ " that 1 indicates acceptance of the alternative hypothesis that GARCH effects exist, (2) "pValue" that is the significance level at which this test rejects the null hypothesis, and (3) "GARCHstat" that indicates GARCH test statistic. Table 1 represents the results of applying the LM test [18] to the contourlet transform of Peppers image (eight directional subbands in the finest scale). This table confirms the existence of two dimensional heteroscedasticity in contourlet coefficients. It should be mentioned that similar results have been obtained for other test images but because of limited space, only the results of peppers image have been reported.

Moreover, the compatibility between the histograms of contourlet coefficients and the 2D-GARCH model is examined. The histograms of two contourlet subbands of Peppers image and the histograms of the best fitted 2D-GARCH model are presented in Fig. 1. To compare the efficiency of 2D-GARCH model with other statistical models, the best fitted Gaussian and Generalized Gaussian (GG) distributions have been plotted in Fig. 1, too. This figure confirms that 2D-GARCH model provides a better compatibility to the data.

\section{Proposed Watermarking Scheme}

As mentioned before, watermarking schemes consist of two main steps: watermark insertion and watermark detection. In the following, we describe these steps for the proposed watermarking method. 
Table 1. Results of two dimensional [18] hypothesis tests for the presence of 2DGARCH effect in the eight contourlet subbands of the finest scale for the Peppers image.

\begin{tabular}{l|l|l|l}
\hline & H & pValue & GARCHstat \\
\hline Subband 1 & 1 & 0 & $1.0166 \mathrm{e}+004$ \\
\hline Subband 2 & 1 & 0 & $2.1819 \mathrm{e}+004$ \\
\hline Subband 3 & 1 & 0 & $7.1432 \mathrm{e}+003$ \\
\hline Subband 4 & 1 & 0 & $6.8305 \mathrm{e}+003$ \\
\hline Subband 5 & 1 & 0 & $1.2155 \mathrm{e}+004$ \\
\hline Subband 6 & 1 & 0 & $6.9759 \mathrm{e}+003$ \\
\hline Subband 7 & 1 & 0 & $2.5357 \mathrm{e}+004$ \\
\hline Subband 8 & 1 & 0 & $8.1015 \mathrm{e}+003$ \\
\hline
\end{tabular}
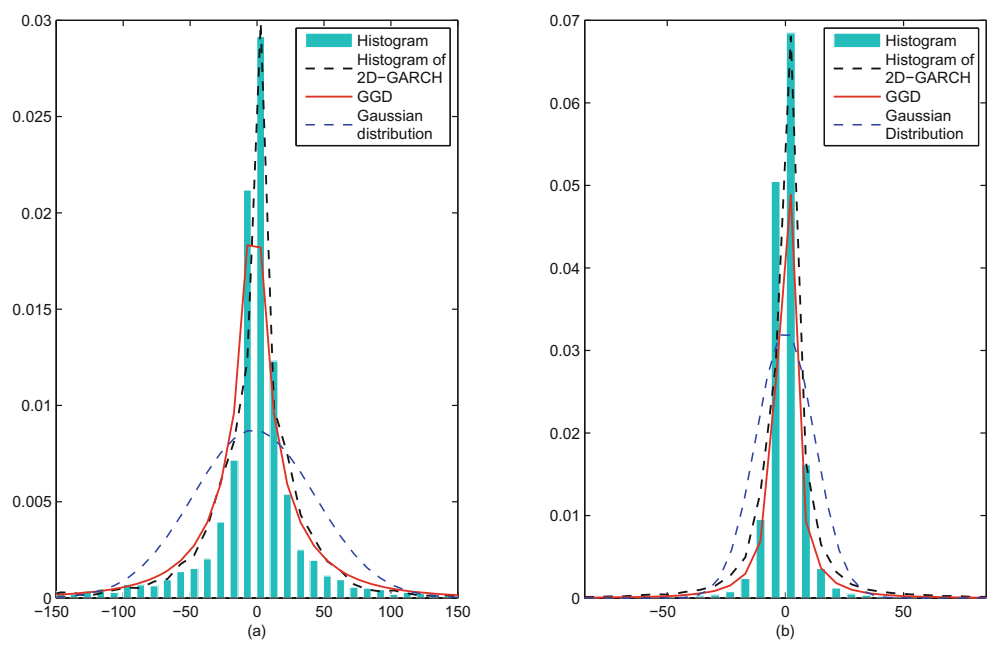

Fig. 1. Histograms of the contourlet coefficients for Peppers image: (a) directional subband in the first pyramidal level (b) directional subband in the third pyramidal level

\subsection{Watermark Insertion}

To embed the watermark into an original image, first, the contourlet transform is applied to the image. Then, to increase the imperceptibility and robustness, the subband with the highest energy is selected to embed the watermark [4]. Additive spread spectrum approach is used to insert the watermark into the selected subband. Let $\mathbf{C}_{\mathbf{x}}=\left\{C_{x}(i, j) \mid i=1, \ldots, M, j=1, \ldots, N\right\}$ denotes the selected subband of the original image. Bold types denote two dimensional vectors. The watermark is embedded into the selected subband using the following formula:

$$
C_{y}(i, j)=C_{x}(i, j)+\alpha s(i, j)
$$

where $\mathbf{C}_{\mathbf{y}}=\left\{C_{y}(i, j) \mid i=1, \ldots, M, j=1, \ldots, N\right\}$ and $\mathbf{s}=\{s(i, j) \mid i=1, \ldots, M$, $j=1, \ldots, N\}$ denote the watermarked contourlet subband and the watermark 
sequence, respectively. $\mathbf{s}$ is a bipolar watermark sequence taking the values -1 and 1 with the equal probability. To obtain $\mathbf{s}$, we use a pseudorandom sequence (PRS) generator with an initial state depends on the value of a secret key. $\gamma$ is the embedding power factor that adjusts the watermark to document ratio (WDR) [19]. After embedding the watermark into the selected contourlet subband, inverse contourlet transform is applied to obtain the watermarked image.

\subsection{Watermark Detection}

To detect the watermark from a given image, contourlet transform is applied to it and the subband with the highest energy is selected. Then, watermark detector should examine the existence of a known watermark in the related contourlet subband $[4,19]$. Due to (6), we can formulate the additive watermark detection in the contourlet domain (the most energetic subband) as the binary hypothesis test:

$$
\begin{aligned}
& \mathcal{H}_{0}: C_{y}(i, j)=C_{x}(i, j) \\
& \mathcal{H}_{1}: C_{y}(i, j)=C_{x}(i, j)+\alpha s(i, j)
\end{aligned}
$$

The above hypothesis test verifies the existence of the given watermark sequence $s(i, j)$ in the contourlet coefficients of the given image $C_{y}(i, j)$. The log-likelihood ratio test is a good choice as a binary hypothesis test for watermark detection because an LLRT based detector maximizes the probability of detection $P_{D}$ (deciding $\mathcal{H}_{1}$ when $\mathcal{H}_{1}$ is true) for a given probability of false-alarm $P_{F A}$ (deciding $\mathcal{H}_{1}$ when $\mathcal{H}_{0}$ is true). So, we design an LLRT based watermark detector and we have:

$$
\log \left\{\Lambda\left(\mathbf{C}_{\mathbf{y}}\right)\right\}=\log \left\{\frac{p\left(\mathbf{C}_{\mathbf{y}} \mid \mathcal{H}_{1}\right)}{p\left(\mathbf{C}_{\mathbf{y}} \mid \mathcal{H}_{0}\right)}\right\} \underset{0}{\underset{0}{\gtrless}} \underset{T}{1} T
$$

where $p\left(\mathbf{C}_{\mathbf{y}} \mid \mathcal{H}_{0}\right)$ and $p\left(\mathbf{C}_{\mathbf{y}} \mid \mathcal{H}_{1}\right)$ denote the probability density functions (pdfs) of $\mathbf{C}_{\mathbf{y}}$ under the conditions $\mathcal{H}_{0}$ and $\mathcal{H}_{1}$, respectively. $T$ is the threshold that controls $P_{D}$ and $P_{F A}$. To obtain an optimal watermark detector using (9), an accurate statistical model of the contourlet coefficients is crucial. In Sect.2.3, the compatibility between 2D-GARCH and contourlet coefficients has been studied and confirmed. So, we design an LLRT based watermark detector using 2D-GARCH model. Suppose the contourlet coefficients of the original image $\mathbf{C}_{\mathbf{x}}$ follow 2D$\operatorname{GARCH}\left(p_{1}, p_{2}, q_{1}, q_{2}\right)$ with the parameters $\left\{\alpha_{0}, \alpha_{01}, \cdots, \alpha_{q_{1} q_{2}}, \beta_{01}, \cdots, \beta_{p_{1} p_{2}}\right\}$. Using (5), (7), and (8), the log-likelihood ratio given in (9) can be written as:

$$
\begin{aligned}
\log \left\{\Lambda\left(\mathbf{C}_{\mathbf{y}}\right)\right\} & =\log \frac{\prod_{i j \in \Phi} p\left(C_{y}(i, j)-\alpha s(i, j) \mid \psi(i, j)\right)}{\prod_{i j \in \Phi} p\left(C_{y}(i, j) \mid \psi(i, j)\right)} \\
& =\log \frac{\prod_{i j \in \Phi} \frac{1}{\sqrt{2 \pi h(i, j)}} \exp \left(\frac{-\left(C_{y}(i, j)-\alpha s(i, j)\right)^{2}}{2 h(i, j)}\right)}{\prod_{i j \in \Phi} \frac{1}{\sqrt{2 \pi h(i, j)}} \exp \left(\frac{-C_{y}(i, j)^{2}}{2 h(i, j)}\right)}
\end{aligned}
$$


where $\psi(i, j)$ and $\Phi$ are as defined in Sect. 2.2. Substituting $h(i, j)$ from (2) in (11) and applying some simplifications, the log-likelihood ratio can be formulated as:

$$
\begin{aligned}
\log \left\{\Lambda\left(\mathbf{C}_{\mathbf{y}}\right)\right\} & =\sum_{i j \in \Phi} \log \sqrt{\frac{\sum_{k \ell \in \Omega_{1}} \alpha_{k \ell} C_{y}(i-k, j-\ell)^{2}+\sum_{k \ell \in \Omega_{2}} \beta_{k \ell} h(i-k, j-\ell)}{\sum_{k \ell \in \Omega_{1}} \alpha_{k \ell}\left(C_{y}(i-k, j-\ell)-w(i-k, j-\ell)\right)^{2}+\sum_{k \ell \in \Omega_{2}} \beta_{k \ell} h(i-k, j-\ell)}} \\
& +\sum_{i j \in \Phi}\left[\frac{C_{y}(i, j)^{2}}{2 \alpha_{0}+\sum_{k \ell \in \Omega_{1}} \alpha_{k \ell} C_{y}(i-k, j-\ell)^{2}+\sum_{k \ell \in \Omega_{2}} \beta_{k \ell} h(i-k, j-\ell)}\right. \\
& \left.+\frac{-\left(C_{y}(i, j)-w_{i j}\right)^{2}}{2 \alpha_{0}+\sum_{k \ell \in \Omega_{1}} \alpha_{k \ell}\left(C_{y}(i-k, j-\ell)-w(i-k, j-\ell)\right)^{2}+\sum_{k \ell \in \Omega_{2}} \beta_{k \ell} h(i-k, j-\ell)}\right]
\end{aligned}
$$

\section{Experimental Results}

In this section, we report the results of simulations. In the proposed method, for multiscale decomposition, 9-7 biorthogonal filters with two levels of pyramidal decomposition are used and in the multi directional decomposition stage, PKVA ladder filters are employed. We carried out extensive simulations on a large number of images but according to the space limitations, we report the results of two $512 \times 512$ grayscale representative images, namely, Peppers and Living room. To examine the statistical significance of our results, we used 50 natural images and report the averaged results. Also, we compare the performance of proposed watermark detector (CT-GARCH) with two other detectors: (1) contourlet domain generalized Gaussian based detector (CT-GG). It should be mentioned that using generalized Gaussian distribution for the contourlet coefficients has been proposed in some papers such as [13]. (2) wavelet domain 2D-GARCH based detector (WT-GARCH) [3]. For 2D-GARCH based detectors, $2 \mathrm{D}-\mathrm{GARCH}(1,1,1,1)$ is used.

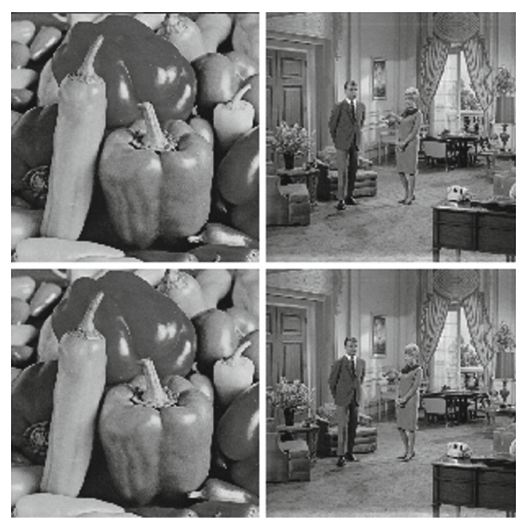

Fig. 2. Test images: "Peppers", and "Living Room" up to down: Original images, and watermarked images using the proposed scheme with $\mathrm{WDR}=-50 \mathrm{~dB}$

Figure 2 shows two test images and the watermarked version of them with $W D R=-50 \mathrm{~dB}$. This figure confirms the imperceptibility of the proposed 
watermarking scheme. To evaluate the performance of watermark detectors, receiver operating characteristic (ROC) curves that are plots of probabilities of detection $P_{D}$ in terms of probabilities of false alarm $P_{F A}$ are used.

First, we study the performance of watermark detectors with out any attacks. Figure 3 presents the ROCs of the contourlet domain 2D-GARCH based detector (CT-GARCH), contourlet domain generalized Gaussian based detector (CTGG), and wavelet domain 2D-GARCH based detector (WT-GARCH) for two test images. It can be inderstood from this figure that the proposed detector provides the highest probability of detection for any chosen value of the false alarm.
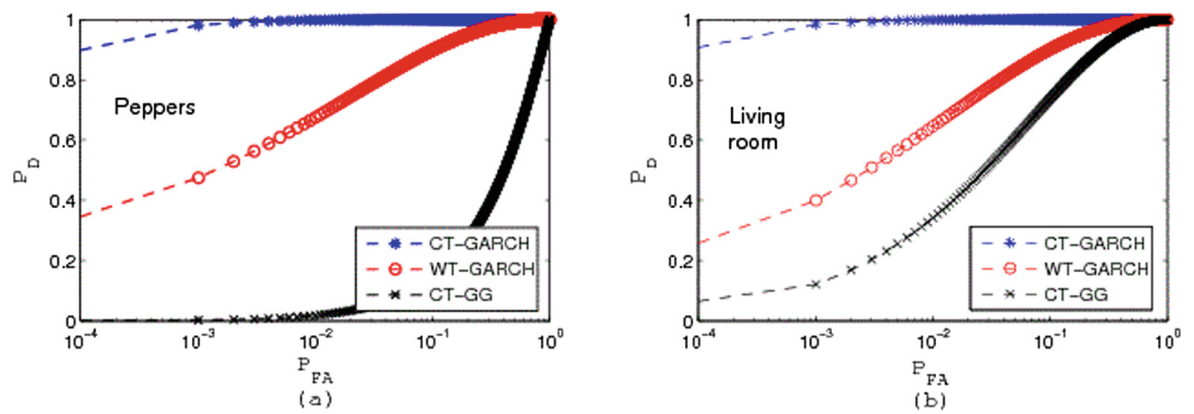

Fig. 3. ROC of the three statistical detectors. Test images are (a) Peppers, and (b) Living Room. (WDR $=-50 \mathrm{~dB}$ )

In the following, we study the performance of proposed watermark detector (CT-GARCH) and also CT-GG and WT-GARCH detectors under some standard attacks, i.e., JPEG compression, median and Gaussian filtering, scaling, and a combinational attack (combination of the Gaussian filtering (window size $=5 \times 5)$ and additive white Gaussian noise $($ AWGN) attacks $(\operatorname{std}=10)$ ). To quantitatively study the detection performance, we use the area under the ROC curve (AUROC) $[2,3,7]$. Table 2 reports the AUROC results of the CTGARCH, CT-GG, and WT-GARCH detectors under these types of attack for $\mathrm{WDR}=-50 \mathrm{~dB}$. Scaling attack has been applied with scaling factor 0.75 . The size of window for median and Gaussian filtering is $5 \times 5$. It is clear from Table 2 that the CT-GARCH detector outperforms the other detectors under all types of attacks.

Finally, to study the statistical significance of the reported results, we perform the robustness tests on 50 natural images. Table 3 reports the averaged AUROC results over 50 images that confirms the higher performance of the proposed method (CT-GARCH) compared with CT-GG and WT-GARCH. 
Table 2. AUROC results under JPEG compression, Scaling, Median filtering, Gaussian filtering and combination of Gaussian filtering and AWGN attacks (WDR $=-50 \mathrm{~dB}$ )

\begin{tabular}{l|l|l|l}
\hline JPEG compression attack $(\mathrm{QF}=60)$ & \multicolumn{1}{l}{} \\
\hline Image & CT-GG detector & WT-GARCH detector & CT-GARCH detector \\
\hline Peppers & 0.7887 & 0.8512 & 0.9814 \\
\hline Living room & 0.7527 & 0.9294 & 0.7527 \\
\hline Scaling attack (scaling factor $=0.75)$ & 0.9920 \\
\hline Peppers & 0.7877 & 0.7516 & 0.9986 \\
\hline Living room & 0.7578 & 0.8335 & \\
\hline Median filtering attack $(5 \times 5)$ & 0.9971 \\
\hline Peppers & 0.8237 & 0.8037 & 1.0000 \\
\hline Living room & 0.9994 & 0.8820 & 1.0000 \\
\hline Gaussian filtering attack $(5 \times 5)$ & 1.0000 \\
\hline Peppers & 0.9439 & 0.8565 & \\
\hline Living room & 0.9269 & 0.8808 & 0.9649 \\
\hline Gaussian Filtering $(5 \times 5)+$ AWGN attacks & 0.9936 \\
\hline Peppers & 0.8864 & 0.7773 & \\
\hline Living room & 0.8299 & 0.7268 & \\
\hline
\end{tabular}

Table 3. Average AUROC results for 50 natural images under different types of attacks $(\mathrm{WDR}=-50 \mathrm{~dB})$

\begin{tabular}{l|l|l|l}
\hline Attack type & CT-GG detector & WT-GARCH detector & CT-GARCH detector \\
\hline Compression $(\mathrm{QF}=60)$ & 0.8463 & 0.8454 & 0.9320 \\
\hline Scaling $(\mathrm{SF}=0.75)$ & 0.7863 & 0.7982 & 0.9654 \\
\hline Median filtering $5 \times 5$ & 0.9324 & 0.8864 & 0.9828 \\
\hline Gaussian filtering $5 \times 5$ & 0.9288 & 0.8705 & 0.9951 \\
\hline Gaussian filtering + AWGN & 0.7536 & 0.7585 & 0.9708 \\
\hline
\end{tabular}

\section{Conclusion}

A novel contourlet domain watermark detector has been proposed in this paper. Watermark is additively embedded into the contourlet subband with the highest energy. Watermark detection is formulated as a binary hypothesis test and the optimal detector can be achieved using LRT. Contourlet domain LRT is based on statistical modeling of contourlet coefficients. Greater the precision of the distribution of contourlet coefficients used in the detector results in the higher the reliability of detection of the watermark. For this purpose, statistical properties of contourlet coefficients have been studied and it has been demonstrated that contourlet coefficients are heteroscedastic. Since none of the previously proposed statistical models for contourlet coefficients can capture the heteroscadsticity, 2D-GARCH model has been proposed for contourlet coefficients and 
its compatibility with contourlet coefficients has been confirmed. Consequently, LRT based contourlet domain watermark detector based on using 2D-GARCH model has been designed. Simulation results demonstrate the high efficiency of proposed method.

\section{References}

1. Cox, I.J., Miller, M.L., Bloom, J.A., Fridrich, J., Kolker, T.: Digital Watermarking and Steganography, 2nd edn. Morgan Kaufmann Publishers, Burlington (2008)

2. Rabizadeh, M., Amirmazlaghani, M., Ahmadian-Attari, M.: A New detector for contourlet domain multiplicative image watermarking using Bessel $\mathrm{K}$ form distribution. J. Vis. Commun. Image Represent. 40, 324-334 (2016)

3. Amirmazlaghani, M.: Additive watermark detection in wavelet domain using 2DGARCH model. Inf. Sci. 370, 1-17 (2016)

4. Sadreazami, H.R., Ahmad, M.O., Swamy, M.N.S.: A study of multiplicative watermark detection in the contourlet domain using alpha-stable distributions. IEEE Trans. Image Process. 23(10), 4348-4360 (2014)

5. Amirmazlaghani, M., Rezghi, M., Amindavar, H.: A novel robust scaling image watermarking scheme based on Gaussian Mixture Model. Expert Syst. Appl. 42, 1960-1971 (2015)

6. Sadreazami, H., Ahmad, M.O., Swamy, M.N.S.: Multiplicative water- mark decoder in contourlet domain using the normal inverse Gaussian distribution. IEEE Trans. Multi. 18(2), 196-207 (2016)

7. Bian, Y., Liang, S.: Locally optimal detection of image watermarks in the wavelet domain using Bessel K Form distribution. IEEE Trans. Image Process. 22(6), 2372$2384(2013)$

8. Okman, O.E., Akar, G.B.: Quantization index modulation-based image watermarking using digital holography. J. Opt. Soc. Am. 24(1), 243-252 (2007)

9. Cheng, Q., Huang, T.S.: Robust optimum detection of transform domain multiplicative watermarks. IEEE Trans. Sig. Process. 51(4), 906-924 (2003)

10. Do, M.N., Vetterli, M.: The contourlet transform: an efficient directional multiresolution image representation. IEEE Trans. Image Process. 14(12), 2091-2106 (2005)

11. Song, H., Yu, S., Yang, X., Song, L., Wang, C.: Contourlet-based image adaptive watermarking. Sig. Process.: Image Commun. 23(3), 162-178 (2008)

12. Jayalakshmi, M., Merchant, S.N., Desai, U.B.: Blind watermarking in contourlet domain with improved detection. In: Proceedings of the Intelligent Information Hiding and Multimedia Signal Processing (IIH-MSP), Pasadena, CA, USA (2006)

13. Qu, H., Peng, Y.: Contourlet coefficient modeling with generalized Gaussian distribution and application. In: Proceedings of the International Conference Audio, Language and Image Processing (ICALIP), July 2008

14. Akhaee, M.A., Sahraeian, S.M.E., Marvasti, F.: Contourlet-based image watermarking using optimum detector in a noisy environment. IEEE Trans. Image Process. 19(4), 967-980 (2010)

15. Amirmazlaghani, M., Amindavar, H., Moghaddamjoo, A.R.: Speckle suppression in SAR images using the 2-D GARCH model. IEEE Trans. Image Process. 18(2), 250-259 (2009)

16. Bollerslev, T.: Generalized autoregressive conditional heteroscedasticity. J. Econom. 31, 307-327 (1986) 
17. Amirmazlaghani, M., Amindavar, H.: Statistical modeling and denoising WignerVille distribution. Digital Sig. Process. 23(2), 506-513 (2013)

18. Amirmazlaghani, M., Amindavar, H.: A novel sparse method for despeckling SAR images. IEEE Trans. Geosci. Remote Sens. 50(12), 5024-5032 (2012)

19. Mahbubur Rahman, S.M., Omair Ahmad, M., Swamy, M.N.S.: A new statistical detector for dwt-based additive image watermarking using the GaussHermite expansion. IEEE Trans. Image Process. 18(8), 1782-1796 (2009) 